\title{
Effects of high intensity interval training on exercise capacity in people with cystic fibrosis: study protocol for a randomised controlled trial
}

Abbey Sawyer ${ }^{1,2,3}$, Vinicius Cavalheri ${ }^{1,2}$, Sue Jenkins ${ }^{1,2,3}$, Jamie Wood ${ }^{1,2,3}$, Nola Cecins ${ }^{2,3}$, Bhajan Singh $^{4,5,6}$ and Kylie Hill ${ }^{1,3^{*}}$

\begin{abstract}
Background: In people with cystic fibrosis (CF), higher exercise capacity is associated with better health-related quality of life (HRQoL), reduced risk of hospitalisation for a respiratory infection and survival. Therefore, optimisation of exercise capacity is an important treatment goal. The Australian and New Zealand clinical practice guidelines recommend that people with CF complete 30 to 60 min of moderate intensity aerobic exercise on most days of the week. This recommendation can be difficult to achieve by people with CF because of time constraints, and intolerable breathlessness and muscle fatigue during continuous exercise. In contrast, a low-volume, high intensity interval training (HIIT) program may be a more achievable and efficient training method to improve exercise capacity in people with CF.
\end{abstract}

Methods: A randomised controlled trial will be undertaken. Forty people with CF (aged $\geq 15$ years) will be randomly allocated, on a 1:1 ratio, to either the experimental or control group. Regardless of their group allocation, all participants will be asked to continue with their usual daily treatment for the study duration. Those in the experimental group will complete 8 weeks of thrice weekly HIIT on a cycle ergometer. Those in the control group will receive weekly contact with the investigators. The primary outcome of this study is exercise capacity. Secondary outcomes are HRQoL, exercise self-efficacy, feelings of anxiety, depression and enjoyment. These outcomes will be recorded at baseline (i.e. prior to randomisation) and following the 8week intervention period. The study will also report other outcomes of the HIIT program (cardiovascular responses, symptom response, post-exercise muscle soreness and tolerance) and behaviour change techniques such as reinforcement, feedback and goal setting, used during the HIIT program.

Discussion: This study will determine the effects of 8-weeks of supervised, low-volume HIIT, completed on a cycle ergometer on measures of exercise capacity, HRQoL, exercise self-efficacy, feelings of anxiety, depression and enjoyment. If effective, this type of training could be an attractive alternative to traditional continuous training because it may be more achievable and time efficient.

Trial registration: Australian and New Zealand Clinical Trials Registry (ANZCTR):12617001271392 (04/09/2017).

Keywords: Cystic fibrosis, Exercise, High intensity interval training

\footnotetext{
* Correspondence: K.Hill@curtin.edu.au

${ }^{1}$ School of Physiotherapy and Exercise Science, Faculty of Health Science,

Curtin University, GPO Box U1987, Perth, WA 6845, Australia

${ }^{3}$ Institute for Respiratory Health, Perth, WA, Australia

Full list of author information is available at the end of the article
}

(c) The Author(s). 2018 Open Access This article is distributed under the terms of the Creative Commons Attribution 4.0 International License (http://creativecommons.org/licenses/by/4.0/), which permits unrestricted use, distribution, and reproduction in any medium, provided you give appropriate credit to the original author(s) and the source, provide a link to the Creative Commons license, and indicate if changes were made. The Creative Commons Public Domain Dedication waiver (http://creativecommons.org/publicdomain/zero/1.0/) applies to the data made available in this article, unless otherwise stated. 


\section{Background}

Cystic fibrosis (CF) is a genetic disease which predominantly affects Caucasians [1]. Several organs are affected by $\mathrm{CF}$ and premature death, primarily due to respiratory failure, is inevitable [1]. Advancements in multidisciplinary care over the past 50 years have resulted in a rise in median life expectancy from 1 year in 1938 [2, 3], 10 years in 1966, 28 years in 1989 [4] to now over 40 years of age [5]. These advancements in multidisciplinary care come at the cost of a high daily treatment burden. That is, the medical, nutritional and physiotherapy regimens for people with CF are time consuming and can take up to $4 \mathrm{~h}$ each day [6], which need to be accommodated with the usual demands of life, such as work, study and family commitments [7].

Compared to their healthy counterparts, important markers of exercise capacity are reduced in people with CF $[8,9]$. The cause of such reduction is multifactorial and includes decrements in lung function, peripheral muscle deconditioning which are both likely to contribute to severe breathlessness during exercise [8]. Additionally, people with CF may have impaired cardiac and vascular function [10-12]. In this population, higher exercise capacity, for example the peak rate of oxygen uptake $\left(\mathrm{VO}_{2 \text { peak }}\right)$, is associated with better health-related quality of life (HRQoL) $[13,14]$, reduced risk of hospitalisation due a respiratory infection [15] and survival [16, 17]. Given this relationship, improving exercise capacity is an important treatment goal in this population [18].

Markers of exercise capacity can be improved in people with CF by undertaking 'traditional' moderate intensity, continuous, aerobic exercise training [19-22]. While the optimal method of exercise training in this population requires further investigation [23], the Australian and New Zealand clinical practice guidelines recommend that people with CF complete 30 to $60 \mathrm{~min}$ of moderate intensity exercise on most days of the week [24]. However, in people with CF, this type of exercise training is burdensome, adding to the already high daily treatment burden, and may not be the most appropriate option due to several factors, including a 'lack of time' [25] and intolerable symptoms of breathlessness and muscle fatigue experienced during continuous exercise [26]. Furthermore, oxygen desaturation is more likely during moderate intensity continuous exercise [27], and this type of training may augment inflammation in this population compared to interval training [28]. An alternative training approach, such as low-volume high intensity interval training (HIIT), may be a more achievable and efficient method to optimise exercise capacity in this population [29].

High intensity interval training consists of short periods of high intensity exercise ('work'), interrupted by periods of lower intensity exercise ('rest') [30]. The 'rest' period allows for partial recovery of symptoms such as breathlessness and muscle fatigue, and therefore offers the opportunity to optimise the training intensity achieved during the 'work' periods [31, 32]. In healthy adults [32] and people with chronic obstructive pulmonary disease (COPD) [33], HIIT is well tolerated and offers similar gains in exercise capacity to continuous training, with fewer symptoms of breathlessness and muscle fatigue [34, 35], and lower training time [36] or work [37] per session. Additionally, HIIT has been reported to be more enjoyable than other modes of exercise training [38]. Preliminary data suggest that HIIT is feasible in people with CF [26, 27]. However, the effectiveness of low-volume HIIT to improve exercise capacity in this population has not been investigated in a randomised controlled trial (RCT).

The primary aim of this RCT is, in people with CF aged $\geq 15$ years, to determine the effects of an 8-week supervised low-volume HIIT program on exercise capacity (primary outcome), and HRQoL, exercise self-efficacy, feelings of anxiety, depression and enjoyment. As a secondary aim, in those allocated to receive HIIT, this study will also report on: (i) the proportion of participants who develop post-exercise quadriceps femoris muscle soreness each week during the 8-week HIIT program as well as the severity of this symptom; (ii) the participant tolerance of the 8-week supervised HIIT program; (iii) the cardiorespiratory and symptom responses elicited during the HIIT sessions throughout the 8-week program and; (iv) the behaviour change techniques (BCTs) employed throughout the program. If the HIIT program is shown to be effective at improving exercise capacity, the implementation of such a time efficient program will result in a lower treatment burden, compared to undertaking moderate intensity continuous exercise and therefore may be more readily incorporated into the daily routine of people with CF.

\section{Methods}

This is a prospective, single-blinded RCT. The study has been approved by the relevant ethics committees and has been registered with the Australian and New Zealand Clinical Trials Registry (ANZCTR) (12617001271392, 04/ 09/2017). Participant recruitment will commence in September 2017. The study will be reported in accordance with the Consolidated Standards of Reporting Trials (CONSORT) statement [39]. The study flow diagram is presented in Fig. 1.

\section{Recruitment and consent}

Potential participants will be identified and recruited from scheduled outpatient clinic appointments at the adult (Sir Charles Gairdner Hospital [SCGH]) and children's (Perth 


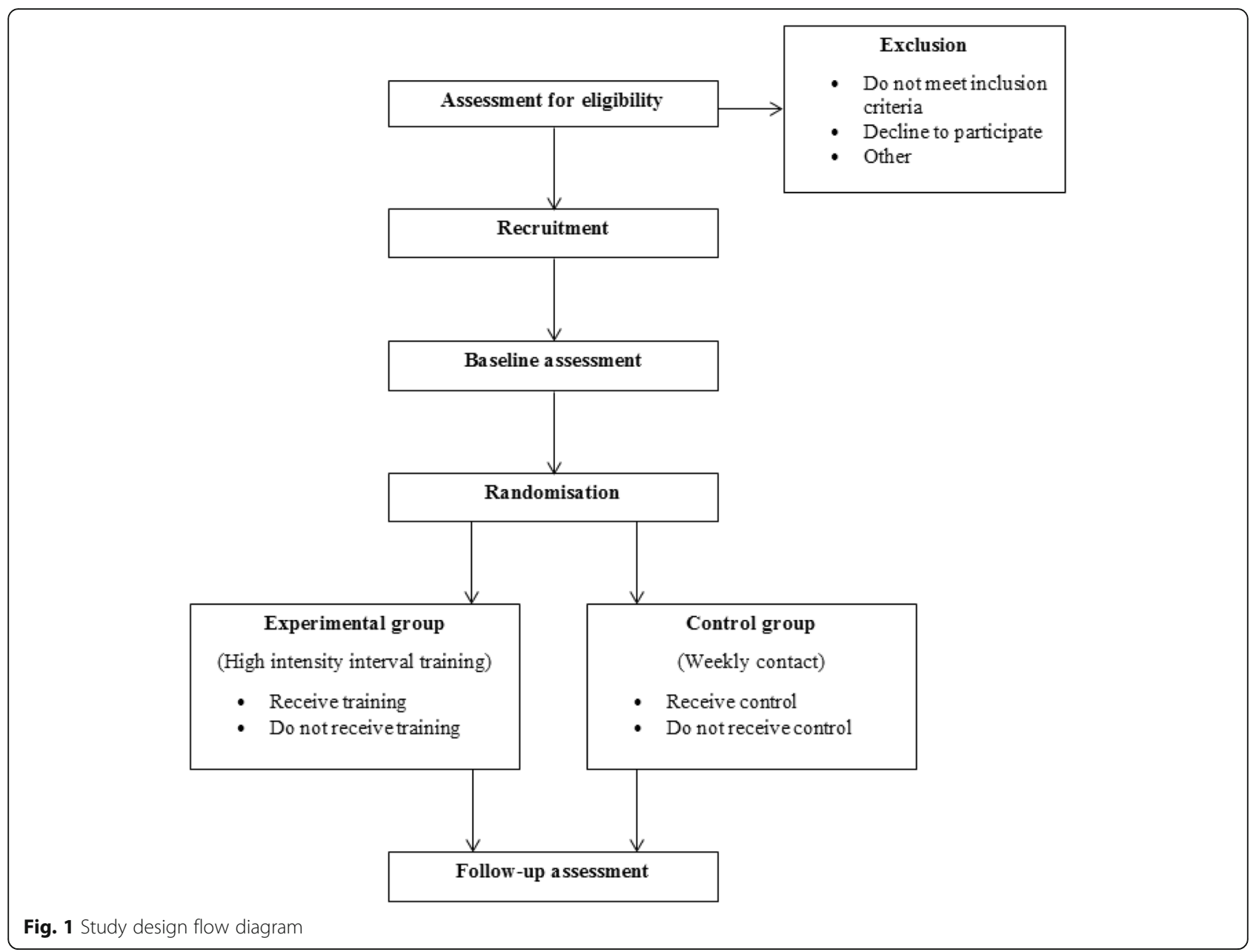

Children's Hospital ([PCH]) CF centres. Potential participants will be provided with an information sheet summarising the study and will receive a phone call within $48 \mathrm{~h}$ of their clinic visit to discuss their willingness to participate. At the beginning of the first study visit, written informed consent will be obtained from participants or assent if the participant is under 18 years of age.

\section{Inclusion and exclusion criteria}

Males and females with CF will be invited to participate in this study if they: (i) are aged $\geq 15$ years and; (ii) have a body mass index (BMI) $>16 \mathrm{~kg} / \mathrm{m}^{2}$. Exclusion criteria will comprise: (i) current or recent (within the previous 4 weeks) exacerbation of $\mathrm{CF}$ which required oral or intravenous antibiotics; (ii) a co-morbid condition that would impact on the ability to undertake a maximal incremental exercise capacity test; (iii) poorly controlled diabetes as deemed by their treating endocrinologist; (iv) previous lung transplant or current listing for lung transplantation; (v) participation in exercise at a moderate intensity two or more times per week for the previous 3 months and; (vi) the inability to provide written informed consent due to a cognitive impairment or being unable to understand English. If a potential participant has cystic fibrosis-related diabetes, they will be included pending approval from the treating endocrinologist, irrespective of the treatment they are on.

\section{Randomisation and allocation concealment}

Participants will be randomly allocated, on a 1:1 ratio, to either the experimental or control group. Concealment of the allocation sequence will be ensured by using a central randomisation service (the National Health and Medical Research Council randomisation service). A minimisation algorithm will be used to stratify for site of recruitment (i.e. SCGH or $\mathrm{PCH}$ ), spirometry (i.e. mild $\left[\mathrm{FEV}_{1} \geq 70 \%\right.$ predicted], moderate $\left[\mathrm{FEV}_{1}\right.$ 40 to $69 \%$ predicted] or severe $\left[\mathrm{FEV}_{1} \leq 39 \%\right.$ predicted]) and the use (or not) of Ivacaftor, which is a medication that improves lung function, weight, HRQoL and reduces the rate of exacerbation [40]. 


\section{Assessment period}

Both prior to randomisation (i.e.baseline) and following the intervention period (i.e. follow-up), participants will complete assessments over two non-consecutive days; assessment day one and assessment day two. Each assessment visit will last $1.5 \mathrm{~h}$, and both visits will be completed within 2 weeks. During both the baseline and follow-up assessments, descriptive variables will be recorded related to age, gender, height, weight, genotype and spirometry (Medgraphics USB Spirometer, MCG Diagnostics, Minnesota, USA). Measures related to the primary aim will be collected in both groups during the baseline and follow-up assessment. This will comprise exercise capacity, HRQoL, exercise self-efficacy, feelings of anxiety, depression and enjoyment. The follow-up assessments of these outcomes will be completed by an assessor who is blinded to group allocation. Measures related to the secondary aim will be collected in the experimental group only and will comprise post-exercise muscle soreness (measured weekly), tolerance (measured at every HIIT session) and cardiorespiratory and symptom responses to HIIT (measured at week 1, week 4 and week 8 of the HIIT program) will be measured throughout the 8-week period. Details of these measurements are provided below.

\section{Assessment day one}

On the first assessment day each participant will complete a ramp-based cycle ergometry test on an electronically-braked cycle ergometer (Ergoselect 100; Ergoline, Bitz, Germany). The test will commence with 1 min of rest, followed by 1 min of unloaded cycling. Thereafter, a 'continuous ramp' protocol will be used to progressively increase the work rate until the participant is unable to continue due to intolerable symptoms (i.e. symptom limitation). The magnitude of change in work rate will be individualised based on the participant's age and pre-existing level of fitness (as subjectively reported by the participant), with the aim of achieving symptom limitation in 8 to $12 \mathrm{~min}$ [41]. Throughout the test, participants will be asked to cycle at a cadence of $60 \mathrm{rpm}$. Breath by breath measurements will be collected of minute ventilation, breathing pattern, rate of oxygen consumption $\left(\mathrm{VO}_{2}\right)$ and rate of expired carbon dioxide production $\left(\mathrm{VCO}_{2}\right)$ (Medgraphics $\mathrm{CardioO}_{2}$; Medical Graphics Corporation). Measures of heart rate (HR) and oxygen saturation $\left(\mathrm{SpO}_{2}\right)$ will be continuously monitored and recorded using a 12 lead electrocardiogram and an ear probe attached to a pulse oximeter (Ohmeda Biox 3700 e ear probe, Colorado, USA), respectively. Blood pressure will be measured every 2 min using an automated blood pressure (BP) machine (Tango M2;
Suntech, North Carolina, USA) with a BP cuff connected to the participants' right arm. Measures of breathlessness and muscle fatigue will be recorded each minute using a modified Borg scale [42]. The peak rate of oxygen uptake $\left(\mathrm{VO}_{2 \text { peak }}\right)$ will be defined as the average $\mathrm{VO}_{2}$ during the final $30 \mathrm{~s}$ of the test [43].

Following completion of the ramp-based cycle ergometry test, the participants will be asked to complete two questionnaires: (i) the Cystic Fibrosis Questionnaire- Revised (CFQ-R) [44, 45] which takes approximately $15 \mathrm{~min}$ to complete and is a valid and reliable questionnaire for use in people with CF [44, 45], and; (ii) the Barriers Self-efficacy Scale (BARSE) [46], which takes approximately $5 \mathrm{~min}$ to complete and is a valid and reliable tool in healthy people [46].

\section{Assessment day two}

On a separate, non-consecutive day, the constant work rate test will be performed in order to establish time to symptom limitation $\left(\mathrm{T}_{\text {lim }}\right)$ (primary outcome). This test will be conducted using identical equipment and measurements as described for the ramp-based cycle-ergometry test. Participants will be asked to complete a 1-min warm-up of unloaded cycling after which the work rate will be increased to $80 \%$ of the $\mathrm{W}_{\max }$ [48]. An intensity of $80 \%$ of the $W_{\text {max }}$ has been chosen as this has been demonstrated to be feasible in people with CF [48]. In addition, at this cycling intensity, more than half of people with chronic respiratory disease (CF or COPD) achieve a $\mathrm{T}_{\lim }$ of between 8 and $11 \mathrm{~min}$; a test duration which is most responsive to change following the intervention period $[49,50]$. During the baseline assessment, if a participant does not demonstrate signs of symptom limitation at $10 \mathrm{~min}$, then the test will be terminated and, following a seated rest of $30 \mathrm{~min}$, the test will be repeated at a higher intensity $[43,50]$. During the follow-up assessment, the constant work rate test will be completed at the highest work rate used during the baseline assessment. In the follow-up assessment, the constant work rate test will be terminated at $20 \mathrm{~min}$ for participants who do not reach symptom limitation earlier and $\mathrm{T}_{\lim }$ will be recorded as $20 \mathrm{~min}$.

Following completion of the constant work rate exercise test, participants will be asked to complete three questionnaires; (i) the Alfred Wellness Score for CF (AweScore-CF) [51], which takes approximately 2 min to complete and is a reliable tool for use in people with CF [51]; (ii) the Hospital Anxiety and Depression Scale (HADS) [52], which takes approximately $10 \mathrm{~min}$ to complete, is a valid, reliable and responsive measure and has been widely used in people with CF [52], and; (iii) the Physical Activity Enjoyment Scale (PACES), which is a valid and reliable tool previously used in healthy people and takes approximately 5 min to complete [53]. 


\section{Intervention period}

Regardless of group allocation, throughout the duration of the study, participants will continue usual care. This includes medication, nutritional support, airway clearance regimens and attendance at a multidisciplinary outpatient CF clinic, which occur quarterly, or more frequently if clinically indicated. In both groups, usual care for each participant will be recorded weekly throughout the intervention period. To do so, participants will be asked if there have been any changes to their treatment in the preceding week.

\section{Experimental group}

Participants allocated to the experimental group will be asked to complete an 8-week cycling-based HIIT program. Each training session will involve a 2-min active 'warm up' at 15 to $20 \mathrm{~W}$, followed by a 30-s 'work' phase and 30-s 'rest' period, repeated six times. The work to rest periods will be followed by a 2 -min active 'cool down' period at 15 to $20 \mathrm{~W}$. Therefore, the total training time per session, inclusive of rest periods, will be $10 \mathrm{~min}$. Each session will be supervised by a physiotherapist who is trained in the management of people with CF. To minimise the onset of post-exercise muscle soreness and optimise adherence, the training program will commence with a 'lead in' phase which will involve only two sessions of HIT in weeks 1 and 2. Thereafter, between weeks 3 and 8 , HIIT will be undertaken three times a week (i.e. total 22 sessions over 8 weeks). The training intensity will be prescribed using measurements of maximum work rate $\left(\mathrm{W}_{\max }\right)$ achieved during the ramp-based cycle ergometry test completed during the baseline assessments. Specifically, the first training session will be prescribed at $60 \%$ of $\mathrm{W}_{\max }$, with the goal of achieving a training intensity equal to $80 \%$ of $\mathrm{W}_{\max }$ during the fourth training session (i.e. the end of week 2). Thereafter, training intensity will be increased as rapidly as symptoms of breathlessness and muscle fatigue permit. Training will be completed at one of two sites, with the participant permitted to choose the site that is most convenient for them. The model of exercise bike used for the HIIT sessions will be identical across all training sites (Orbit Eco Generator Interval Bike OEB2002, Perth, Australia). Only a single participant will be permitted to use the exercise room on any day, with stringent hand hygiene and cleaning procedures adhered to at all times to reduce the risk of cross contamination between participants [54]. The intervention will be conducted and reported in accordance with the TIDieR checklist [55] and CERT guidelines [56, 57].

If a participant in the experimental group reports the onset of symptoms indicative of an exacerbation of $\mathrm{CF}$ (i.e. increased sputum volume, changes in the characteristics of sputum, blood present in the sputum, increased cough, pain from coughing, new wheeze or increased wheeze, new or increased chest tightness, shortness of breath or difficulty breathing, increased fatigue or lethargy, fever, loss of appetite or weight, sinus pain or tenderness) [58], they will be referred to their respective $\mathrm{CF}$ team for medical review. If an exacerbation is diagnosed by the treating $\mathrm{CF}$ team, the participant will be invited to continue participating in HIIT sessions once deemed to be medically stable by a CF clinician. Medical stability has been defined as being afebrile, having resting $\mathrm{BP}, \mathrm{HR}$ and $\mathrm{SpO}_{2}$ within limits of the participants' baseline values and/or deemed stable by the treating CF clinician. In the event of missed attendance to HIIT sessions, the training program will be extended by a maximum of 2 weeks so that the participant will have 10 weeks to complete the 22 HIIT sessions.

\section{Measurements related to the secondary aim (collected in the experimental group only)}

Post-exercise muscle soreness Participants in the experimental group will be asked if they experienced any post-exercise quadriceps femoris muscle soreness whilst completing a 'sit to stand' $24 \mathrm{~h}$ following the first training session of each week. Those who report experiencing post-exercise muscle soreness will be asked to rate its severity using a Visual Analogue Scale (VAS) [59].

Tolerance The level of participant attendance and completion of the HIIT sessions will be recorded throughout the 8-week period. Oxygen saturation $\left(\mathrm{SpO}_{2}\right)$ at rest and nadir $\mathrm{SpO}_{2}$ will be recorded during each training session. Adverse events will be monitored throughout the HIIT program. These will be categorised as minor, if they are transient and self-limiting events (i.e. breathlessness without significant oxygen desaturation $[<4 \%$ from the participant's pre-exercise $\mathrm{SpO}_{2}$ ], muscle or general fatigue) or major events if they require the participant to cease training during any given session or necessitate medical assistance (i.e. breathlessness with significant oxygen desaturation $[\geq 4 \%$ from the participants pre-exercise $\mathrm{SpO}_{2}$ ], pain, vasovagal events or haemoptysis).

Cardiorespiratory and symptom responses During weeks 1,4 and 8 of the training period, participants in the experimental group will be asked to complete one of two or three (week 3 onwards) HIIT sessions in the same laboratory used to conduct the ramp-based cycle ergometry test. During these sessions, measurements will be collected of minute ventilation, breathing pattern, $\mathrm{VO}_{2}$ and $\mathrm{VCO}_{2}, \mathrm{HR}, \mathrm{SpO}_{2}$ and $\mathrm{BP}$. Breathlessness and muscle fatigue will be assessed using the modified Borg scale [42]. 
Behaviour change techniques The HIIT sessions will be audio recorded over the course of the 8-week program. The purpose of this will be to allow for qualitative analysis and reporting of BCTs [60], as recommended by the CONSORT guidelines [61]. Behaviour change techniques are defined as the active component of an intervention that targets a specific behaviour. These techniques can be used alone, or in combination with another technique. Of note, to be considered a BCT, the intervention needs to be observable, able to be replicated, and designed to alter existing or stimulate new behaviour [60]. Some examples of BCTs are reinforcement, self-monitoring, feedback, problem solving, graded tasks and reward. The BCTs will be reported in accordance with the Taxonomy (v1) by Michie et al. [60]. All HIIT sessions will be audio recorded, unless the participant 'opts-out'. This audio recording process will also allow for fidelity checking of the sessions if required by the relevant ethics committee.

\section{Control group}

Participants allocated to the control group will be contacted once a week by a physiotherapist to discuss changes to their symptoms, healthcare utilisation and participation in exercise over the preceding week. Participants will be allowed to choose the way in which this contact is made; phone calls, texts or emails. If a participant allocated to the control group reports any symptoms that are suggestive of an exacerbation, they will be referred to the CF team for medical review.

\section{Statistical analyses}

The results of this study will be analysed according to the intention-to-treat principle using Statistical Package for the Social Sciences (SPSS) (Version 22, SPSS, Chicago, IL, USA). The distribution of data will be checked using frequency histograms. A $p$ value of < 0.05 will be considered statistically significant. Audio recording of the HIIT sessions will be analysed using NVivo software.

To address differences between the experimental and control group, between-group differences in all outcomes (e.g. $\mathrm{T}_{\text {lim }}, \mathrm{VO}_{2 \text { peak }}, \mathrm{HRQ}$ oL, exercise self-efficacy, feelings of anxiety, depression and enjoyment) will be assessed using linear mixed models. Baseline measures will be used as a covariate and group allocations will be used as a fixed factor. Total in work done (i.e. training dose) will be considered in the analyses by entering this parameter as a random factor in the mixed effects model. To address the proportion of participants who develop post-exercise muscle soreness, the severity of post-exercise muscle soreness in those who develop it over the 8 week intervention period and tolerance of the HIIT program, descriptive statistics, such as frequency, mean and standard deviation (SD) or median and interquartile range will be used. To explore differences in measures of cardiorespiratory and symptom responses throughout the HIIT program, an analysis of variance (parametric) or a Friedman's test (non-parametric data) will be used.

\section{Sample size calculations}

Constant power tests have been widely used to demonstrate increases in exercise capacity following a period of exercise training (i.e. pulmonary rehabilitation) [43, 47], are also more likely to reflect performance of activities of daily living [62], as they are performed at a moderate intensity, compared to tests of peak exercise capacity $[22,43]$ and when conducted at $80 \%$ of the $\mathrm{W}_{\max }$ are demonstrated to be highly repeatable in people with CF [63]. For these reasons, the sample size calculation for this study has been based on the measurements of $\mathrm{T}_{\text {lim }}$ during a constant work rate test, conducted at $80 \%$ of $\mathrm{W}_{\max }$ [43]. There is limited information pertaining to the minimal clinically important difference (MCID) for the $\mathrm{T}_{\text {lim }}$ in people with CF. However, in people with COPD, the MCID for $\mathrm{T}_{\text {lim }}$ during a constant work rate test has been proposed to be $100 \mathrm{~s}$ [43]. In order to detect a between-group difference of $100 \mathrm{~s}$ (MCID for $\mathrm{T}_{\text {lim }}$ in COPD), with a SD of $99 \mathrm{~s}$ (largest of the $2 \mathrm{SDs}$ reported for $\mathrm{T}_{\text {lim }}$ in a CF population that will be similar to that recruited in the study described in this proposal [48]) $(\alpha=0.05$ and $1-\beta$ $=0.8)$ a sample size of 16 per group ( $n=32$ in total) is required. This sample size has been inflated by $20 \%$ to account for possible loss to follow-up. Therefore, the recruitment target for this study will be 40 .

\section{Discussion}

This study will be the first RCT to evaluate the effects of HIIT on exercise capacity, HRQoL, exercise self-efficacy, feelings of anxiety, depression and enjoyment in people with CF. If $30 \mathrm{~min}$ of HIIT (i.e. three 10 min sessions) per week is demonstrated to increase exercise capacity, HRQoL, exercise self-efficacy and enjoyment, it will represent a more achievable and efficient method of exercise and thereby will overcome the barrier of 'lack of time' [25]. This study will create an initial, yet strong evidence base for HIIT in people with $\mathrm{CF}$ and has the ability to influence clinical practice.

\footnotetext{
Abbreviations

ANZCTR: Australian New Zealand clinical trials registry; AweScore-CF: Alfred wellness score for cystic fibrosis; BARES: Barriers self-efficacy scale; BMI: Body mass index; BP: Blood pressure; CF: Cystic fibrosis; CFQ-R: Cystic fibrosis questionnaire revised; CONSORT: Consolidated standards of reporting trials; COPD: Chronic obstructive pulmonary disease; FEV ${ }_{1}$ : Forced expiratory volume in one second; HADS: Hospital anxiety and depression scale; HIIT: High intensity interval training; HR: Heart rate; HRQoL: Health-related quality of life; MCID: Minimal clinically important difference; PACES: Physical activity enjoyment scale; PCH: Perth children's hospital; RCT: Randomised controlled trial; SCGH: Sir Charles Gairdner Hospital; SD: Standard deviation;
} 
$\mathrm{SpO}_{2}$ : Oxygen saturation measured using a pulse oximeter; SPSS: Statistical package for the social sciences; $T_{\text {lim }}$ : Time to symptom limitation; VAS: Visual analogue scale; $\mathrm{VCO}_{2}$ : Rate of expired carbon dioxide production; $\mathrm{VO}_{2}$ : Rate of oxygen consumption; $\mathrm{VO}_{2 \text { peak: }}$ : Peak rate of oxygen uptake; $\mathrm{W}_{\text {max }}$ : Maximal work rate

\section{Acknowledgements}

The authors would like to thank the respiratory scientists at the Department of Pulmonary Physiology and Sleep Medicine (SCGH), the staff of the adult CF Centre at SCGH (Dr Siobhain Mulrennan, Dr. Anna Tai and Ms. Sue Morey) and Dr. Andre Schultz of the paediatric CF centre at PCH. The authors would like to acknowledge the Conquer Cystic Fibrosis Research Program, the Institute for Respiratory Health and the Australian Cystic Fibrosis Research Trust (Top-Up Scholarship) for financial support in the form of a scholarship for AS

\section{Funding}

This work was supported by Curtin University and Sir Charles Gairdner Hospital (Physiotherapy Department start-up funding and the Research Advisory Committee in the form of a grant). AS is supported by scholarships which have been made available through the Institute for Respiratory Health (Conquer Cystic Fibrosis Research Program), the Australian Government Research Training Program Scholarship and Cystic Fibrosis Australia (Australian Cystic Fibrosis Research Trust Top-Up Scholarship). VC is funded by a Cancer Council Western Australia Postdoctoral Research Fellowship.

\section{Availability of data and materials}

The datasets used and/or analysed during the current study are available from the corresponding author on reasonable request.

\section{Authors' contributions}

AS, VC, KH, SJ, JW and NC designed and planned the study. AS is responsible for recruitment, data collection and supervision of the HIIT program, and this will be overseen by VC, KH and SJ for fidelity purposes. JW will assist with recruitment and collection of follow-up assessment questionnaire data. BS has assisted with planning the exercise tests and will oversee the collection process and analysis of exercise data. All members have reviewed the draft versions of this paper and have read and approved the final manuscript.

\section{Ethics approval and consent to participate}

This protocol is approved by the Human Research Ethics Committee at Sir Charles Gairdner Hospital, on behalf of Sir Charles Gairdner Hospital and Perth Children's Hospital (RGS 0000000065) with reciprocal approval from Curtin University (HRE2017-0651). Written informed consent will be obtained from each participant

\section{Consent for publication}

Not applicable.

\section{Competing interests}

The authors declare that they have no competing interests.

\section{Publisher's Note}

Springer Nature remains neutral with regard to jurisdictional claims in published maps and institutional affiliations.

\section{Author details}

${ }^{1}$ School of Physiotherapy and Exercise Science, Faculty of Health Science, Curtin University, GPO Box U1987, Perth, WA 6845, Australia. ${ }^{2}$ Physiotherapy Department, Sir Charles Gairdner Hospital, Perth, WA, Australia. ${ }^{3}$ Institute for Respiratory Health, Perth, WA, Australia. ${ }^{4}$ Department of Pulmonary Physiology and Sleep Medicine, Sir Charles Gairdner Hospital, Perth, WA, Australia. ${ }^{5}$ West Australian Sleep Disorders Research Institute, Nedlands, WA, Australia. ${ }^{6}$ Faculty of Science, University of Western Australia, Crawley, WA, Australia.
Received: 7 February 2018 Accepted: 26 October 2018

Published online: 06 November 2018

\section{References}

1. Bell SC, Bye PT, Cooper PJ, Martin AJ, McKay KO, Robinson PJ, Ryan GF, Sims GC. Cystic fibrosis in Australia, 2009: results from a data registry. Med J Aus. 2011;195(7):396-400

2. Shoemaker MJ, Hurt $\mathrm{H}$, Arndt L. The evidence regarding exercise training in the management of cystic fibrosis: a systematic review. Cardiopulm Phys Ther J. 2008;19(3):75-83.

3. Orenstein DW, Winnie GB, Altman H. Cystic fibrosis: a 2002 update. J Pediatr. 2002;140:156-64.

4. Orenstein D. Cystic fibrosis. Respir Care. 1991;36:746-54.

5. Keogh RH, Szczesniak R, Taylor-Robinson D, Bilton D. Up-to-date and projected estimates of survival for people with cystic fibrosis using baseline characteristics: a longitudinal study using UK patient registry data. J Cyst Fibros. 2018;17(2):218-27.

6. Sawicki GS, Sellers DE, Robinson WM. High treatment burden in adults with cystic fibrosis: challenges to disease self-management. J Cyst Fibros. 2009; 8(2):91-6.

7. Boyle MP. So many drugs, so little time: the future challenge of cystic fibrosis care. Chest. 2003;123(1):3-5.

8. Troosters T, Langer D, Vrijsen B, Segers J, Wouters K, Janssens W, Gosselink R, Decramer M, Dupont L. Skeletal muscle weakness, exercise tolerance and physical activity in adults with cystic fibrosis. Eur Respir J. 2009;33(1):99-106.

9. Saynor ZL, Barker RA, Oades JP, Williams CA. Impaired aerobic function in patients with cystic fibrosis during ramp exercise. Med Sci Sports Exerc. 2014:46(12):2271-8.

10. Hull JH, Garrod R, Ho TB, Knight RK, Cockcroft JR, Shale DJ, Bolton CE. Increased augmentation index in patients with cystic fibrosis. Eur Respir J. 2009;34(6):1322-8.

11. Hull JH, Ansley L, Bolton CE, Sharman JE, Knight RK, Cockcroft JR, Shale DJ, Garrod R. The effect of exercise on large artery haemodynamics in cystic fibrosis. J Cyst Fibros. 2011;10(2):121-7.

12. Van Iterson EH, Wheatley CM, Baker SE, Morgan WJ, Snyder EM. The relationship between cardiac hemodynamics and exercise tolerance in cystic fibrosis. Heart and lung: J acute. Crit Care. 2016;45(3):283-90.

13. de Jong W, Kaptein AA, van der Schans CP, Mannes GP, van Aalderen W, Grevink RG, Koëter GH. Quality of life in patients with cystic fibrosis. Pediatr Pulmonol. 1997;23(2):95-100.

14. Orenstein DM, Nixon PA, Ross EA, Kaplan RM. The quality of well-being in cystic fibrosis. Chest. 1989;95(2):344-7.

15. Perez M, Groeneveld IF, Santana-Sosa E, Fiuza-Luces C, Gonzalez-Saiz L, VillaAsensi JR, Lopez-Mojares LM, Rubio M, Lucia A. Aerobic fitness is associated with lower risk of hospitalization in children with cystic fibrosis. Pediatr Pulmonol. 2014;49(7):641-9.

16. Nixon PA, Orenstein DM, Kelsey SF, Doershuk CF. The prognostic value of exercise testing in patients with cystic fibrosis. N Engl J Med. 1992;327(25): $1785-8$.

17. Pianosi P, Leblanc J, Almudevar A. Peak oxygen uptake and mortality in children with cystic fibrosis. Thorax. 2005;60(1):50-4.

18. Yankaskas JR, Marshall BC, Sufian B, Simon RH, Rodman D. Cystic fibrosis adult care: consensus conference report. Chest. 2004;125:1-39.

19. Schneiderman JE, Wilkes DL, Atenafu EG, Nguyen T, Wells GD, Alarie N, Tullis E, Lands LC, Coates AL, Corey M, Ratjen F. Longitudinal relationship between physical activity and lung health in patients with cystic fibrosis. Eur Respir J. 2014;43(3):817-23.

20. Klijn PH, Oudshoorn A, van der Ent CK, van der Net J, Kimpen JL, Helders PJ. Effects of anaerobic training in children with cystic fibrosis: a randomized controlled study. Chest. 2004;125(4):1299-305.

21. Selvadurai HC, Blimkie CJ, Meyers N, Mellis CM, Cooper PJ, Van Asperen PP. Randomized controlled study of in-hospital exercise training programs in children with cystic fibrosis. Pediatr Pulmonol. 2002;33(3):194-200.

22. Moorcroft AJ, Dodd ME, Morris J, Webb AK. Individualised unsupervised exercise training in adults with cystic fibrosis: a 1 year randomised controlled trial. Thorax. 2004;59(12):1074-80

23. Radtke T, Nolan SJ, Hebestreit H, Kriemler S. Physical exercise training for cystic fibrosis. Paed Resp Rev. 2016;19:42-5.

24. Button BM, Wilson C, Dentice R, Cox NS, Middleton A, Tannenbaum E, Bishop J, Cobb R, Burton K, Wood M. Physiotherapy for cystic fibrosis in 
Australia and New Zealand: a clinical practice guideline. Respirology. 2016; 21(4):656-67.

25. White $D$, Stiller $K$, Haensel N. Adherence of adult cystic fibrosis patients with airway clearance and exercise regimens. J Cyst Fibros. 2007;6(3):163-70.

26. Gruber W, Orenstein DM, Braumann KM, Beneke R. Interval exercise training in cystic fibrosis- effects on exercise capacity in severely affected adults. J Cyst Fibros. 2014;13(1):86-91.

27. Kaltsakas G, Anastasopoulos N, Chynkiamis N, Zeliou P, Karapatoucha V, Kotsifas K, Diamantea F, Inglezos I, Koulouris NG, Vogiatzis I. Effect of high intensity interval exercise rehabilitation in cystic fibrosis. Eur Resp J. 2017;50: OA310.

28. Nguyen T, Obeid J, Ploeger HE, Takken T, Pedder L, Timmons BW. Inflammatory and growth factor response to continuous and intermittent exercise in youth with cystic fibrosis. J Cyst Fibros. 2012;11(2):108-18.

29. Stevens D, Oades PJ, Williams CA. Airflow limitation following cardiopulmonary exercise testing and heavy-intensity intermittent exercise in children with cystic fibrosis. Eur J of Paediatr. 2015;174(2):251-7.

30. Gibala MJ, Little J, MacDonald MJ, Hawley JA. Physiological adaptations to low-volume, high-intensity interval training in health and disease. J Physiol. 2012;590(5):1077-84.

31. Helgerud HK, Wang E, Karlsen T, Berg P, Bjerkaas M, Simonsen T, Helgesen C, Hjorth N, Bach R, Hoff J. Aerobic high-intensity intervals improve $\mathrm{VO}_{2}$ max more than moderate training. Med Sci Sports Exerc. 2007;39(4):665-71.

32. Weston KS, Wisløff U, Coombes JS. Effects of low-volume high-intensity interval training (HIT) on fitness in adults: a meta-analysis of controlled and non-controlled trials. Sports Med. 2014;44(7):1005-17.

33. Beauchamp MK, Nonoyama M, Goldstein RS, Hill K, Dolmage TE, Mathur $\mathrm{S}$, Brooks D. Interval versus continuous training in individuals with chronic obstructive pulmonary disease- a systematic review. Thorax. 2010;65(2):157-64

34. Arnardóttir $\mathrm{RH}$, et al. Interval training compared with continuous training in patients with COPD. Resp Med. 2007;101(6):1196-204.

35. Mador MJ, Krawza M, Alhjhusian A, Khan Al, Shaffer M, Kufel TJ. Interval training versus continuous training in patients with chronic obstructive pulmonary disease. J Cardiopulm Rehabil Prev. 2009;29(2):126-32.

36. Gillen JB, Martin BJ, Maclnnis MJ, Skelly LE, Tarnopolsky MA, Gibala MJ. Twelve weeks of sprint interval training improves indices of cardiometabolic health similar to traditional endurance training despite a five-fold lower exercise volume and time commitment. PLoS One. 2016;11(4):e0154075.

37. Puhan MA, Büsching G, Schünemann HJ, Zaugg C, Frey M. Interval versus continuous high-intensity exercise in chronic obstructive pulmonary disease: a randomized trial. Ann Intern Med. 2006;145(11):816-25.

38. Bartlett JD, Close GL, MacLaren DPM, Gregson W, Drust B, Morton JP. Highintensity interval running is perceived to be more enjoyable than moderate-intensity continuous exercise: implications for exercise adherence. J Sports Sci. 2011;29(6):547-53.

39. Schulz KF, Altman DG, Moher D. CONSORT 2010 statement: updated guidelines for reporting parallel group randomised trials. BMC Med. 2010;8(1):18

40. Ramsey BW, Davies J, McElvaney NG, Tullis E, Bell SC, Dřevínek P, Griese M, McKone E, Wainwright C, Konstan MW. A CFTR potentiator in patients with cystic fibrosis and the G551D mutation. N Engl J Med. 2011;365(18):1663-72.

41. ATS/ACCP statement on cardiopulmonary exercise testing. Am J Respir Crit Care Med. 2003;167(2):211-77.

42. Borg G. Psychophysical bases of perceived exertion. Med Sci Sports Exerc. 1982;14(5):377-81.

43. Puente-Maestu L, Palange P, Casaburi R, Laveneziana P, Maltais F, Neder JA, O'Donnell DE, Onorati P, Porszasz J, Rabinovich R. Use of exercise testing in the evaluation of interventional efficacy: an official ERS statement. Eur Respir J. 2016;47(2):429-60

44. Quittner AL, Buu A, Messer MA, Modi AC, Watrous M. Development and validation of the cystic fibrosis questionnaire in the United States: a healthrelated quality-of-life measure for cystic fibrosis. Chest. 2005;128(4):2347-54.

45. Wenninger K, Aussage P, Wahn U, Staab D. The revised German cystic fibrosis questionnaire: validation of a disease-specific health-related quality of life instrument. Wual Life Res. 2003;12(1):77-85.

46. McAuley E. The role of efficacy cognitions in the prediction of exercise behavior in middle-aged adults. J Behav Med. 1992;15(1):65-88.

47. Porszasz J, Rambod M, van der Vaart H, Rossiter HB, Ma S, Kiledjian R, Casaburi R. Sinusoidal high-intensity exercise does not elicit ventilatory limitation in chronic obstructive pulmonary disease. Exp Physiol. 2013;98(6): $1102-14$.

48. McKone EF, Barry SC, Fitzgerald MX, Gallagher CG. The role of supplemental oxygen during submaximal exercise in patients with cystic fibrosis. Eur Respir J. 2002;20(1):134-42.

49. Borel B, Provencher S, Saey D, Maltais F. Responsiveness of various exercisetesting protocols to therapeutic interventions in COPD. Pulm Med. 2013; 2013:410748.

50. van der Vaart H, Murgatroyd SR, Rossiter HB, Chen C, Casaburi R, Porszasz J. Selecting constant work rates for endurance testing in COPD: the role of the power-duration relationship. Copd. 2014;11(3):267-76.

51. Button BM, Gufler A, Clark D, Mitchell L, Wilson JW. The development of a new quick/easy CF wellness score (Alfred wellness score for CF, "AweScore$\left(F^{\prime \prime}\right)$ to improve delivery of clinical care in the outpatient and inpatient settings suggests patients acclimatise to low lung function. J Cyst Fibros. 2014;13:105.

52. Snaith R. The hospital anxiety and depression scale. Health Qual Life Outcomes. 2003;1:29.

53. Kendzierski D, DeCarlo KJ. Physical activity enjoyment scale: two validation studies. J Sport Exerc Psychol. 1991;13(1):50-64.

54. Saiman L, Siegel JD, LiPuma JJ, Brown RF, Bryson EA, Chambers MJ, Downer VS, Fliege J, Hazle LA, Jain M. Infection prevention and control guideline for cystic fibrosis: 2013 update. Infect Control Hosp Epidemiol. 2014;35(S1):S1-S67.

55. Hoffmann TC, Glasziou PP, Boutron I, Milne R, Perera R, Moher D, Altman DG, Barbour V, Macdonald H, Johnston M. Better reporting of interventions: template for intervention description and replication (TIDieR) checklist and guide. BMJ. 2014;348:1687.

56. Slade SC, Dionne CE, Underwood M, Buchbinder R. Standardised method for reporting exercise programmes: protocol for a modified Delphi study. BMJ Open. 2014;4(12):1514-24.

57. Slade SC, Dionne CE, Underwood M, Buchbinder R, Beck B, Bennell K, Brosseau L, Costa L, Cramp F, Cup E. Consensus on exercise reporting template (CERT): modified delphi study. Phys Ther. 2016;96(10):1514-24.

58. Goss $\mathrm{CH}$, Burns JL. Exacerbations in cystic fibrosis.1: epidemiology and pathogenesis. Thorax. 2007;62(4):360-7.

59. Bijur PE, Silver $E$, Gallagher EJ. Reliability of the visual analog scale for measurement of acute pain. Acad Emerg Med. 2001;8(12):1153-7.

60. Michie S, Richardson M, Johnston M, Abraham C, Francis J, Hardeman W, Eccles MP, Cane J, Wood CE. The behavior change technique taxonomy (v1) of 93 hierarchically clustered techniques: building an international consensus for the reporting of behavior change interventions. Ann Behav Med. 2013;46(1):81-95.

61. Boutron I, Moher D, Altman DG, Schulz KF, Ravaud P. Extending the CONSORT statement to randomized trials of nonpharmacologic treatment: explanation and ElaborationMethods and processes of the CONSORT group. Ann Intern Med. 2008;148(4):295-309.

62. Williams CA, Saynor ZL, Tomlinson OW, Barker AR. Cystic fibrosis and physiological responses to exercise. Expert Rev Respir Med. 2014;8(6): 751-62.

63. Barry SC, Gallagher CG. The repeatability of submaximal endurance exercise testing in cystic fibrosis. Pediatr Pulmonol. 2007:42(1):75-82.

Ready to submit your research? Choose BMC and benefit from:

- fast, convenient online submission

- thorough peer review by experienced researchers in your field

- rapid publication on acceptance

- support for research data, including large and complex data types

- gold Open Access which fosters wider collaboration and increased citations

- maximum visibility for your research: over $100 \mathrm{M}$ website views per year

At $\mathrm{BMC}$, research is always in progress.

Learn more biomedcentral.com/submission 